\title{
Intragenic homogenization and multiple copies of prey-wrapping silk genes in Argiope garden spiders
}

\author{
R Crystal Chaw ${ }^{1 *}$, Yonghui Zhao ${ }^{1}$, Jie Wei ${ }^{1,2}$, Nadia A Ayoub ${ }^{1,3}$, Ryan Allen ${ }^{1}$, Kirmanj Atrushi ${ }^{1}$ and Cheryl Y Hayashi ${ }^{1}$
}

\begin{abstract}
Background: Spider silks are spectacular examples of phenotypic diversity arising from adaptive molecular evolution. An individual spider can produce an array of specialized silks, with the majority of constituent silk proteins encoded by members of the spidroin gene family. Spidroins are dominated by tandem repeats flanked by short, non-repetitive $\mathrm{N}$ - and C-terminal coding regions. The remarkable mechanical properties of spider silks have been largely attributed to the repeat sequences. However, the molecular evolutionary processes acting on spidroin terminal and repetitive regions remain unclear due to a paucity of complete gene sequences and sampling of genetic variation among individuals. To better understand spider silk evolution, we characterize a complete aciniform spidroin gene from an Argiope orb-weaving spider and survey aciniform gene fragments from congeneric individuals.

Results: We present the complete aciniform spidroin (AcSp1) gene from the silver garden spider Argiope argentata (Aar_AcSp1), and document multiple AcSp1 loci in individual genomes of A. argentata and the congeneric A. trifasciata and $A$. aurantia. We find that Aar_AcSp1 repeats have $>98 \%$ pairwise nucleotide identity. By comparing AcSp1 repeat amino acid sequences between Argiope species and with other genera, we identify regions of conservation over vast amounts of evolutionary time. Through a PCR survey of individual A. argentata, A. trifasciata, and A. aurantia genomes, we ascertain that $A c S p 1$ repeats show limited variation between species whereas terminal regions are more divergent. We also find that average $\mathrm{dN} / \mathrm{dS}$ across codons in the $\mathrm{N}$-terminal, repetitive, and C-terminal encoding regions indicate purifying selection that is strongest in the $\mathrm{N}$-terminal region.

Conclusions: Using the complete A. argentata AcSp1 gene and spidroin genetic variation between individuals, this study clarifies some of the molecular evolutionary processes underlying the spectacular mechanical attributes of aciniform silk. It is likely that intragenic concerted evolution and functional constraints on A. argentata AcSp1 repeats result in extreme repeat homogeneity. The maintenance of multiple AcSpl encoding loci in Argiope genomes supports the hypothesis that Argiope spiders require rapid and efficient protein production to support their prolific use of aciniform silk for prey-wrapping and web-decorating. In addition, multiple gene copies may represent the early stages of spidroin diversification.
\end{abstract}

Keywords: AcSp1, Concerted evolution, Intragenic homogenization, Multiple loci, Silk, Spidroin, Spider

\section{Background}

Spider silks are ideal for studying the molecular evolutionary processes that create and maintain adaptive characteristics. An individual spider can produce and use different silk types singly or in combination for specific tasks, with each silk type having mechanical properties well-suited to its function. For example, aciniform silk is used in prey immobilization and egg sac construction [1,2]. The

\footnotetext{
*Correspondence: rcrystal@ucr.edu

'Department of Biology, University of California, 900 University Avenue, Riverside 92507, Riverside, CA, USA

Full list of author information is available at the end of the article
}

mechanical properties of aciniform silk include impressive extensibility and toughness [1], making it excellent for swathing struggling prey because it is easy to stretch but difficult to break. Orb-weaving garden spiders from the genus Argiope are renowned for their use of aciniform silk. Argiope employ many layers of aciniform silk to completely immobilize and envelop their prey (e.g. [3,4]), and Argiope are also a model system for studying the purpose of aciniform-silk web decorations, known as stabilimenta, that have been implicated in predator avoidance, prey attraction, and web stability (for review see [5,6]). 
Of the five fibrous silks spun by the silver garden orbweaver Argiope argentata, aciniform silk is the toughest and one of the most extensible [7]. However, little is known about the evolution of aciniform silk's physical attributes. Spider silk mechanical properties are related to the suite of proteins that compose each silk type. The majority of spider silk proteins, or spidroins (a contraction of "spider-fibroins" [8]), are encoded by members of a single gene family. Spidroins are typically very large $(>200 \mathrm{kDa})$, and are dominated by a series of iterated repeats flanked by short amino (N)- and carboxy (C)terminal regions $[9,10]$. The length, number, and amino acid (aa) composition of the iterated repeats are silktype specific, whereas phylogenetic analyses have shown that aa residues in the $\mathrm{N}$ - and $\mathrm{C}$-terminal regions are more conserved across spidroins [11,12]. Repeat aa sequence corresponds to secondary structures that are partly responsible for silk mechanical properties (e.g. [13-15]), and conservation of the $\mathrm{N}$ - and C-terminal regions [12] and their presence in spun silk fibers suggests an important role in spider silk biology [16-18].

The evolutionary maintenance of spidroin repeat sequences within a silk type and the divergence of those repeat sequences between silk types is central to spider silk function and diversity. Within a particular spidroin, repeat units tend to be highly similar, or homogeneous, in amino acid and nucleotide sequence. The gene encoding aciniform spidroin $(A c S p 1)$ has repeats that are relatively complex among spidroin family members, however, despite this complexity, AcSp1 repeats are also spectacularly homogenized $[1,19]$. A recent analysis of a complete AcSp1 from the western black widow Latrodectus hesperus showed that its repetitive region, like those of other spidroins, is dominated by the amino acids glycine (G), alanine (A), and serine (S) [19]. However, L. hesperus AcSp1 repeats have few or none of the short glycine and alanine-rich subunits, such as GGX, poly-GA, and poly-A, that can be the bulk of other spidroin repeats [9]. Nevertheless, L. hesperus AcSp1 repeats are remarkably homogenized ( $>99 \%$ identity at the nucleotide level [19]). This is consistent with results from a partial length AcSp1 cDNA from the banded garden orb-weaver Argiope trifasciata, which has 14 repeats that are each $600 \mathrm{bp}$ and share $99.9 \%$ identity at the nucleotide level [1].

The high level of AcSp1 repeat homogeneity is frequently attributed to gene conversion or unequal crossing over resulting in intragenic concerted evolution (e.g. $[1,19,20])$. Concerted evolution usually refers to homogenization among gene family members, such as rDNA gene copies [21], but it can also occur within a gene [22,23]. Stabilizing selection alone would maintain protein sequence, resulting in a high level of repeat identity at the aa level. However, the extreme level of homogenization reported for AcSp1 repeats provides evidence for concerted evolution because it exists at both the protein and nucleotide levels $[1,19]$.

In addition to concerted evolution, repeat homogeneity in AcSp1 may be maintained by functional constraints. Recent nuclear magnetic resonance (NMR) studies of AcSp1 repeats from both Nephila antipodiana and $A$. trifasciata delineate different domains in each repeat unit, one domain that is rich in alpha helices and one that is not $[24,25]$. Xu et al. [25] used NMR and dihedral angles from global likelihood estimate (DANGLE) analyses to predict the chemical shift indices of a 199 aa recombinant A. trifasciata AcSp1 repeat. The consensus secondary structure assignments specified that the last quarter of the protein was unstructured, but that the first three-quarters of the repeat contained six major helical regions. Protein structures such as these six alpha helices are considered the foundation for silk mechanical properties (e.g. [25,26]).

Assessing the extent to which a spidroin is homogenized within a single gene or among individuals is difficult because the repetitive region makes it exceptionally challenging to sequence complete spidroin genes. Indeed, partial length sequences that are biased toward the C-terminus greatly dominate the number of published spidroins [12]. Additionally, the evolutionary processes leading to spidroin divergence between species and silk types are often unclear due to a lack of knowledge about spidroin genetic variation among individuals.

Here, we address these issues by presenting a complete spidroin gene from an Argiope spider, the AcSp1 sequence of $A$. argentata (Aar_AcSp1), and by screening for $A c S p 1$ variation among individual $A$. argentata, $A$. trifasciata, and $A$. aurantia spider genomes. Sequencing the full array of $A a r_{-} A c S p 1$ repeats enabled us to test hypotheses of concerted evolution and functional constraints. Based on previous spidroin research, Aar_AcSp1 repeats should be extremely homogenous at the nucleotide and amino acid levels. In addition, amino acid sequences that are predicted to correspond to the structural motifs that contribute to the toughness and extensibility of aciniform silk should be more conserved between Argiope species relative to surrounding regions. Among the surveyed $A$. argentata individuals, we expected Aar_AcSp1 to be a single-copy gene, similar to L. hesperus AcSp1 [19]. Between species, previous research suggests that the spidroin repeats within each silk type are highly conserved, but that the terminal regions show more variation [12], and we hypothesized that Aar_AcSp1 would also follow this pattern.

\section{Results and discussion}

Argiope argentata AcSp1 complete sequence and phylogenetic placement

Despite obtaining 59 AcSp1 cDNA clones, including one that was $>8 \mathrm{~kb}[1]$, a complete Argiope AcSp1 remained 
elusive until the present study. By screening a large-insert genomic DNA library, we sequenced and assembled 18,080 bp of $A$. argentata DNA including a complete open reading frame that is $13,440 \mathrm{bp}$ long and predicted to encode a 4,479 aa A. argentata AcSp1 (Aar_AcSp1; Figure 1; GenBank KJ206620). No introns were detected. The putative protein has a predicted size of $\sim 430 \mathrm{kDa}$, and the most abundant amino acids are serine (22.6\%), alanine (14.4\%) and glycine (13.3\%). Aar_AcSp1 has three regions, a central repetitive region that is flanked by conserved $\mathrm{N}$ - and $\mathrm{C}$-terminal regions. The repetitive region dominates $\sim 90 \%$ of the protein and is composed of 20 iterated repeats (Figure 1). The first 19 repeats are each 204 aa, and the last repeat is 186 aa due to truncation at the end (Figure 1). The length, amino acid composition, and organization of AcSp1 are all consistent with other spidroin family members [9].

Phylogenetic analyses of Aar_AcSp1 N- and C-terminal coding regions with those from other spidroins grouped Aar_AcSp1 with the Latrodectus (widow spider) AcSp1 sequences in a well-supported clade (bootstrap value $=98 \%$; Figure 2). Latrodectus and Argiope are estimated to have diverged from each other 175 million years ago (MYA) [27]. Despite this lengthy time period, the recovery of an AcSp1 clade was consistent with prior studies in which spidroin sequences nearly always grouped based on silk type (e.g. $[9,10])$. The sister group to the AcSp1 clade was TuSp1, tubuliform (egg-case) spidroin, suggesting that these paralogs have a relatively recent common ancestor [19]. Further potential evidence of their shared ancestry is that both of these silk types are used in egg-case construction and both have repeats that are relatively complex compared to other spidroins [28]. In our phylogenetic analysis, a large, weakly supported assemblage of spidroins is sister to the combined AcSp1 and TuSp1 clade. Given the low support, it is unclear which spidroins are most closely related to AcSp1 and TuSp1.

\section{Argiope argentata AcSp1 repeat homogeneity}

As expected, Aar_AcSp1 repeats are complex and spectacularly homogenized. Although glycine, alanine, and serine account for $\sim 50 \%$ of its repetitive region composition, Aar_AcSp1 has few of the glycine/alanine-rich motifs such as GGX, GPG, poly-GA, and poly-A that are dominant in the dragline major ampullate spidroins (MaSp1, MaSp2) from Argiope and other taxa [9]. At the nucleotide level, the average pairwise percent identity between Aar_AcSp1 repeats is an astonishing 98.7\%. Complexity and extreme homogenization are also features of previously described $A c S p 1$ sequences $[1,19]$.

The extreme nucleotide identity of Aar_AcSp1 is consistent with concerted evolution, and cannot be easily explained by codon usage bias. For example, Aar_AcSp1 codon use is strongly influenced by amino acid position within a repeat. In our repeat alignment (Additional file 1: Figure S1), the neighboring alanine codons at nucleotide positions 103-105 and 106-108 are GCC and GCT, respectively. GCCGCT is present in the same relative location in all twenty repeats. Similarly, the glycine codons that appear at nucleotide positions 64-66 and 130-132 also consistently use different codons (GGT and GGA, respectively). The same alternative codons are used at the same exact positions throughout most, if not all, the repeats. Despite a slight skew toward alanine codons that end in adenine (A) or thymine (T) $(55.0 \%$ GCW, W being the IUPAC ambiguity code for A or T; Additional file 2: Table S5), it is difficult to postulate that selective forces acting at the level of codon usage are responsible for the extensive homogeneity of codon positions found throughout the $612 \mathrm{bp}$ Aar_AcSp1 repeat. Concerted evolution that fixes particular codons at particular locations across repeats provides a clearer explanation.

Analyses of the full array of Aar_AcSp1 iterated repeats were also consistent with two concerted evolution predictions. First, ML analysis grouped araneid AcSp1 repeats into well-supported, species-specific clades rather than grouping the repeats across species (Figure 3A). Furthermore, nucleotide pairwise identity within each species averaged $98 \%$, but pairwise identity between Aar_AcSp1 repeats and repeats from other species averaged only $78.5 \%$ (73.6\% vs. Araneus ventricosus, $79.1 \%$ vs. A. trifasciata, and $82.8 \%$ vs. A. amoena). That repeats are more similar within species than between species regardless of intragenic repeat position can be explained by rapid intra-specific spread of genetic variation via unequal crossing over during recombination [22,23].

Second, the average nucleotide pairwise identity of the first and last Aar_AcSp1 repeats to the rest of the array is slightly lower at $96 \%$ and $93 \%$, respectively. Less similar first and last repeats are consistent with some models of concerted evolution [29]. However, araneid AcSp1 first and last repeats still grouped within species-specific clades (Figure 3A), suggesting that these repeat sequences are more homogeneous within a gene than those of previously analyzed spidroins. For example, in an analysis of repetitive units from the flagelliform spidroin (Flag) of the golden orb-weaver Nephila clavipes and the congeneric Nephila inaurata madagascariensis, the first repeats grouped together across species, and the last repeats also formed their own clade. By contrast, the central (not first or last) repeats formed species-specific clades because each repetitive unit was nearly identical within each species yet divergent across species [30]. Longer estimated divergence times between the species in our present study may explain the more thorough homogenization of araneid $A c S p 1$ sequences compared to that of the previously studied Nephila Flag sequences. The estimated divergence time between the Nephila species is 7.4 MYA [31], 


\begin{tabular}{|c|c|c|c|c|c|c|c|c|c|c|c|c|c|c|c|c|c|c|c|c|}
\hline \multicolumn{21}{|c|}{$\begin{array}{l}\text { Argiope argentata AcSp1: 4,479aa } \\
\text { Iterated repeats }\end{array}$} \\
\hline $\mathrm{N}$ & 1 & 2 & 3 & 4 & 5 & 6 & 7 & 8 & 9 & 10 & 11 & 12 & 13 & 14 & 15 & 16 & 17 & 18 & 19 & $20 \mid C$ \\
\hline \multicolumn{7}{|c|}{ Spidroin N-term } & & \multicolumn{9}{|c|}{ Repeat 11 (exemplar repeat) } & \multicolumn{4}{|c|}{ Spidroin C-term } \\
\hline \multirow{2}{*}{\multicolumn{7}{|c|}{$\begin{array}{l}\text { MNWLPTLAFAILLLSVQYDAV } \\
\text { OSASTLSRSPWANPAKAGSLM }\end{array}$}} & \multicolumn{10}{|c|}{ IISRVANAIANTSTLRAVLRRGVS $Q Q$} & \multicolumn{4}{|c|}{ VGLRSGSAASRIR } \\
\hline & \multicolumn{5}{|c|}{ QSASTLSRSPWANPAKAGSLM } & & \multicolumn{10}{|c|}{ TASSVVQRAAOSIASTLGVDGNNLSR } & \multicolumn{4}{|c|}{ QLTSSVTNAVGPN } \\
\hline \multicolumn{8}{|c|}{ NCLMSRIASSNVLPQQDKEDL } & \multicolumn{9}{|c|}{ VALQAISQVPTGSDTSAYAOAFSSA } & \multicolumn{4}{|c|}{ GVDANALARSLQS } \\
\hline \multicolumn{7}{|c|}{ ESIMDTLMSAIKGASAKGKSS } & & \multicolumn{9}{|c|}{ FNAGVLNASNIDTLGSRVLSAVLNGV } & \multicolumn{4}{|c|}{ SFSNLRSSGMSSS } \\
\hline \multicolumn{8}{|c|}{ AAQIQAINMAVASS $\perp A E I V V A$} & \multicolumn{9}{|c|}{ SSAA GLGINVDTGSVQSDISSSSS $F$} & \multicolumn{4}{|c|}{ DAKIEVLLETIVS } \\
\hline \multicolumn{8}{|c|}{ EDAGNQASIAVKTQALTGALG } & \multicolumn{9}{|c|}{ ISTSSSASS SS ASASSTSGAGYG } & \multicolumn{4}{|c|}{ LLQLISNTQIRGV } \\
\hline \multirow{2}{*}{\multicolumn{7}{|c|}{ QCF'QAVMGTVDRKFINEINDL }} & & \multirow{2}{*}{\multicolumn{9}{|c|}{ SGYTGPVGGAQGSASG SS SOT }} & \multirow{2}{*}{\multicolumn{4}{|c|}{$\begin{array}{l}\text { NPATASSVANSAA } \\
\text { RSFELVIA }\end{array}$}} \\
\hline & & & & & & & & & & & & & & & & & & & & \\
\hline
\end{tabular}

Figure 1 Schematic of the protein encoded by the complete Argiope argentata aciniform spidroin 1 gene (GenBank KJ206620). Predicted protein is 4,479 aa. Conserved spidroin N- (orange) and C-terminal (blue) domains (shaded boxes) flank 20 iterated repeats (numbered boxes). Boxes are drawn to scale and standardized to the 204 aa length of the first 19 repeats. Arrows point to corresponding amino acid sequences for each domain. Exemplar repeat 11 has 100\% identity to the majority rule consensus of the repeat sequences. Alanine (red), serine (purple), and glycine (green) are shaded to emphasize the abundance of those amino acids.

whereas Araneus and Argiope are thought to have diverged 30 MYA and within Argiope, 23 MYA between A. argentata and $A$. trifasciata [32].

\section{Functional constraints on AcSp1 repeats}

We predicted that functional constraints would result in greater aa sequence conservation in the portion of each repeat proposed by $\mathrm{Xu}$ et al. to contain six alpha helices [25]. To test this, we first compared known Araneidae repetitive regions. We aligned consensus AcSp1 repeat sequences from three Argiope species (A. argentata, A. trifasciata, A. amoena) and Araneus ventricosus. We then graphed pairwise identities for each aligned position between the $A$. trifasciata repeat sequence and the other species, and plotted it against the predicted $A$. trifasciata domains from Xu et al. [25] (Figure 3B). Using amino acid positions from $\mathrm{Xu}$ et al. [25], the average percent pairwise identity over the 150 aa helix-rich domain was $84.0 \%$, but only $54.1 \%$ over the remaining 49 aa. Xu et al. [25] also noted a major alpha-helical domain from 102-151 aa, encompassing the region denoted as helix 5 and 6 in Figure 3B. Consistent with being structurally important, the average percent identity in this domain was $90.7 \%$. Moreover, our alignment was slightly longer (216 aa) than the A. trifasciata recombinant repeat length (199 aa) due to indels that only appeared in the unstructured region. Notably, in the region from 200-209 aa (our alignment), the $A$. trifasciata repeat has a deletion (Figure 3B). These indels further indicate that the final quarter of AcSp1 repeats is less conserved than the first three-quarters.

To investigate amino acid conservation in the predicted AcSp1 helical regions across greater evolutionary time, we also aligned consensus amino acid AcSp1 repeat sequences from $L$. hesperus and Uloborus diversus to the A. trifasciata repeat from Xu et al. $[19,25]$. Araneidae, represented here by Argiope and Araneus, and Theridiidae, represented by $L$. hesperus, are members of the superfamily Araneoidea, with araneids and theridiids estimated to have last shared a common ancestor $~ 175$ MYA [27]. $U$. diversus is within the Deinopoidea, the sister-group to the Araneoidea. Araneoids and deinopoids diverged from each other 210 MYA [27]. Together, Araneoidea and Deinopoidea compose the Orbiculariae (orb-web weaving spiders and their relatives).

The AcSp1 repeat units from $L$. hesperus and $U$. diversus are almost twice as long as the araneid AcSp1 repeat units. The $L$. hesperus and $U$. diversus repeat units can be further subdivided into two parts that align with each other [19]. We aligned each part from each species (two parts per species) to the A. trifasciata repeat separately. We then calculated the average pairwise percent identity for each comparison and for each of the six putative alpha-helical regions predicted by $\mathrm{Xu}$ et al. ([25]; Additional file 2: Table S6). The overall pairwise identities between $L$. hesperus repeat part 1 and $U$. diversus repeat part 1 with the A. trifasciata repeat was $30 \%$ and $29 \%$, respectively. Of note, the percent pairwise identity between L. hesperus repeat part 1 and the A. trifasciata repeat was $47 \%$ in the $A$. trifasciata region associated with helix 4 , and it was $41 \%$ against $U$. diversus repeat part 1 in the region associated with helix $6.47 \%$ and $41 \%$ were the highest pairwise identity percentages.

Our results strongly support the hypothesis that functional constraints are acting to conserve protein sequence in the repetitive region of $\mathrm{AcSp} 1$. Our comparison of A. trifasciata AcSp1 repeat sequence with that of other 


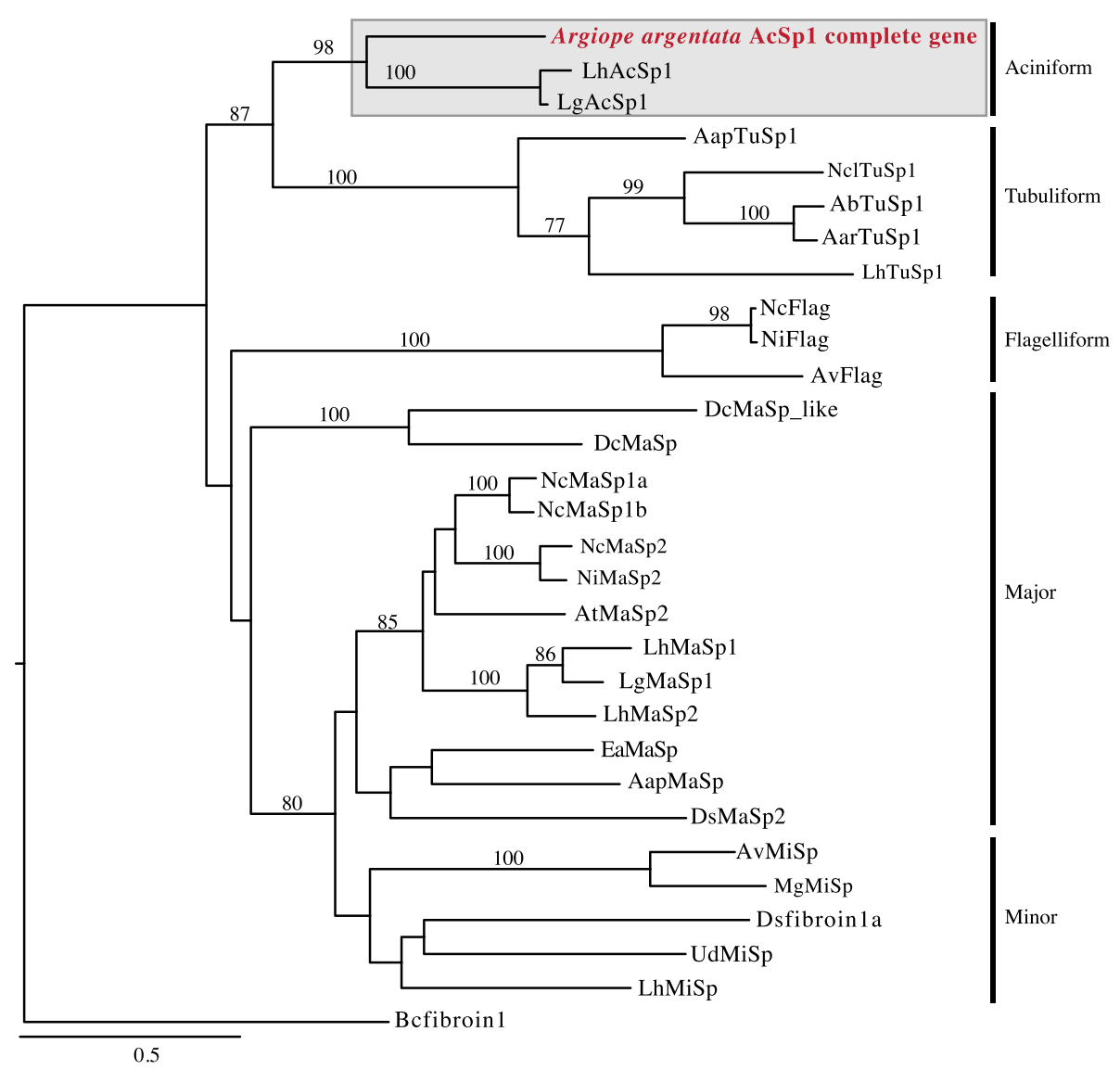

Figure 2 Maximum likelihood tree of concatenated N- and C-terminal coding regions from 29 published spidroins and Aar_AcSp1 from this study (accession numbers in Additional file 2: Table S2). Box highlights aciniform clade, with Argiope argentata AcSp1 further indicated in red. Vertical bars identify clades by silk type. Bootstrap values greater than 70\% are shown. Abbreviations defined in Additional file $2:$ Table S2. Scale bar represents substitutions per site.

araneid species indicates that a specific amino acid sequence is maintained in the predicted helix-rich domain of AcSp1 repeats across Araneidae. In contrast, comparison between the A.trifasciata repeat with part 1 of repeats from $L$. hesperus and $U$. diversus indicates that amino acid sequence in the regions associated with alpha-helices 4 and 6 are the most highly conserved across Orbiculariae. The higher level of conservation in the amino acid sequences corresponding to helices 4 and 6 may indicate that these regions impart the same general function across Orbiculariae whereas the other predicted helical regions of $A$. trifasciata impart functions unique to Araneidae. Sequencing AcSp1 from other genera of Araneidae and other families of Orbiculariae will enable further elucidation of these hypotheses.

To our knowledge, there are no current predictions about the secondary structures of $L$. hesperus or $U$. diversus AcSp1 repeats. It is feasible that, like the AcSp1 domains of $N$. antipodiana and A. trifasciata, L. hesperus and $U$. diversus AcSp1 repeats also feature distinct structural regions. Finally, our analysis may be an underestimation of sequence conservation because it does not include amino acid replacements that are functionally equivalent. However, predicting functional protein similarity is difficult given the extensive physicochemical changes that spider silk undergoes as it is processed from a liquid into dry silk (e.g. [33,34]).

\section{Delineation of ACSp1 variants in individual Argiope spiders}

Spidroin sequence variation between individual spiders is an important source of genetic variation for the evolution of different silk types within and between species. To investigate genetic variation in $A c S p 1$ between individuals of $A$. argentata and the congeneric $A$. trifasciata and $A$. aurantia, we first designed PCR primers targeting the repetitive region of Aar_AcSp1. Amplification of genomic DNA across species and individuals resulted in AcSp1 repeat sequences that did not show intraspecific variation but had significant inter-specific variation (Figure 4A). Intraspecific homogenization of the repeats could be explained by biased PCR amplification of a single repeat type in the repetitive region, however, our results are consistent 

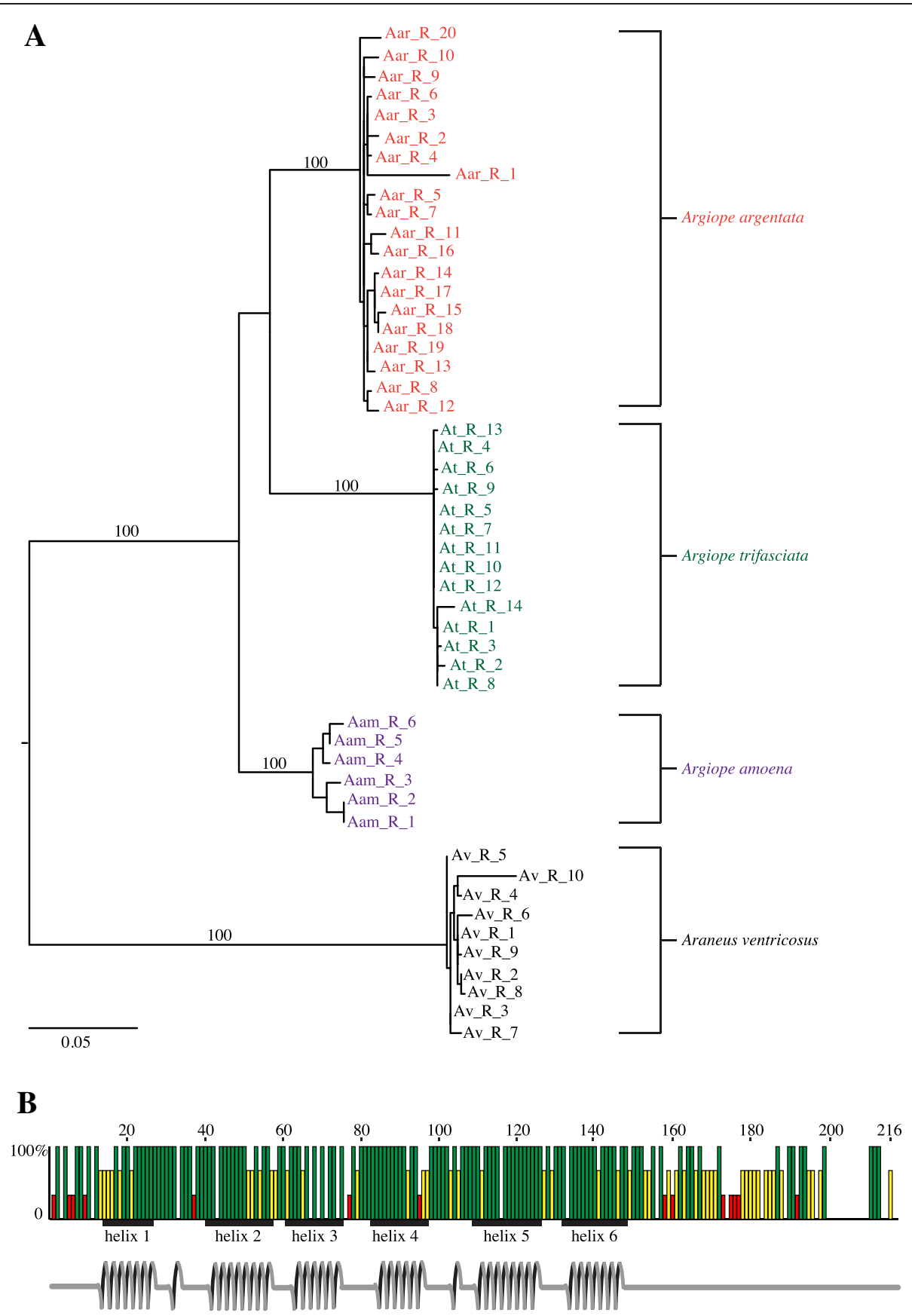

Figure 3 Concerted evolution and selection on the repetitive region of AcSp1. (A) Iterated AcSp1 repeats show intra-specific homogenization in the family Araneidae. Midpoint-rooted maximum likelihood tree of AcSp1 DNA repeats (R) from A. amoena (Aam; purple), A. argentata (Aar; orange), A. trifasciata (At; green), and Araneus ventricosus (Av; black). Repeats are numbered from 5' to 3'. Bootstrap values for species-specific groups are shown. Scale bar indicates substitutions per site. (B) Functional constraint on repeat sequence. Graph of the pairwise identities of consensus AcSp1 repeat sequences from two Argiope species (A. argentata and A. amoena) and Araneus ventricosus to that of Argiope trifasciata. Bars show 100\% (green), 66\% (yellow), or 33\% (red) identity at each position, helical domains found by NMR and DANGLE analyses of A. trifasciata AcSp1 repeat sequence shown in schematic under the graph (helix redrawn from [25]).

with the high degree of repeat sequence conservation in AcSp1 sequences from araneids (Figure 3A) and $L$. hesperus [19].
Next, we designed PCR primers targeting $\mathrm{N}$ - and $\mathrm{C}$ terminal coding regions of Aar_AcSp1. We then amplified the same individual genomic DNAs that were surveyed 

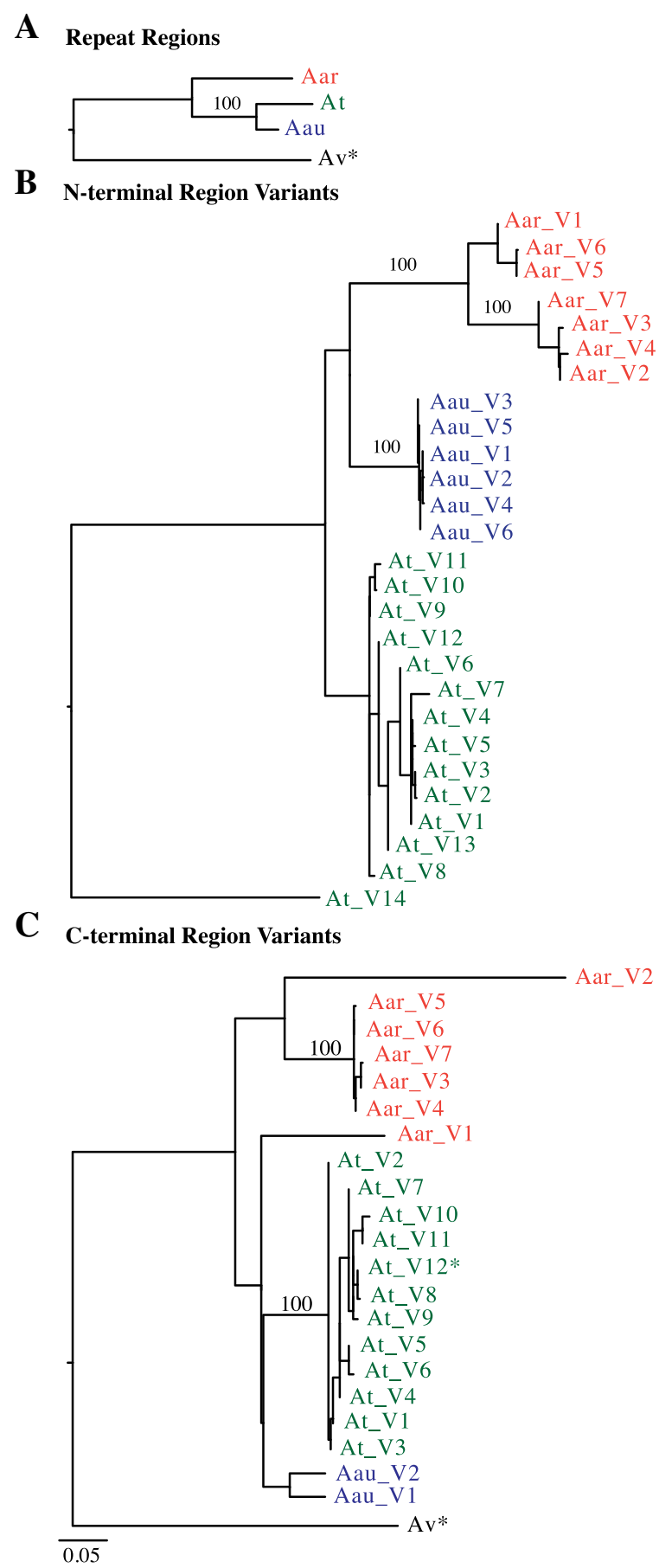
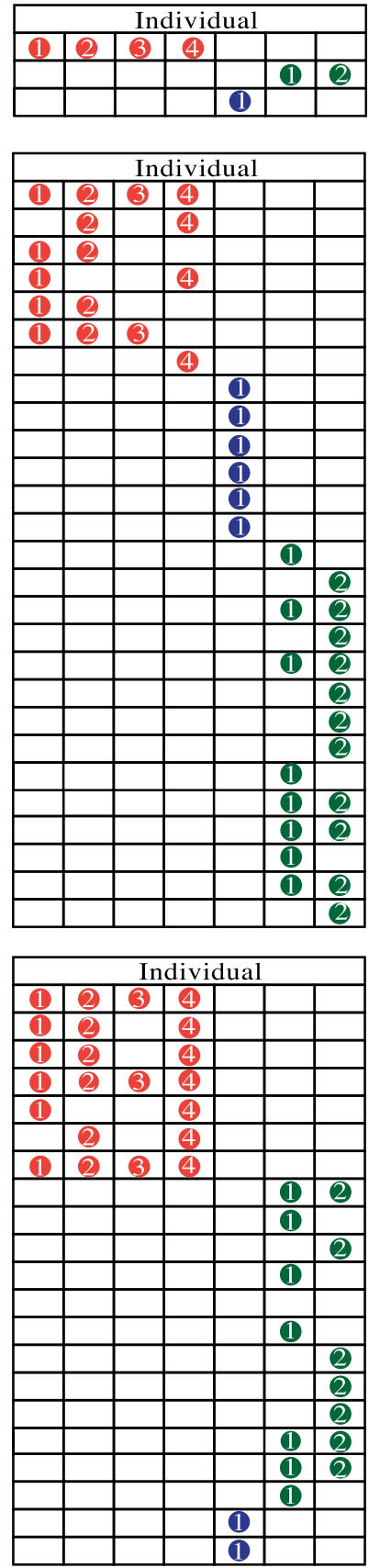

Figure 4 Multiple AcSp1 loci in Argiope. Maximum likelihood nucleotide trees of PCR amplified sequences from the (A) repetitive, (B) N-terminal, and (C) C-terminal coding regions of ACSp1 from three Argiope species, A. argentata (Aarg; orange), A. aurantia (Aau; blue), and A. trifasciata (At; green). Araneus ventricosus ( $\mathrm{Av}$; black) sequence (GenBank HQ008714) was used to root repeat and C-terminal trees. N-terminal tree is midpoint-rooted (B). For each variant, the adjacent table row indicates the status of that variant in individual spiders. Each individual per species was assigned a number that appears in the corresponding row if a variant was detected. Bootstrap values of $100 \%$ are shown. ${ }^{*}$ denotes outgroup or published A. trifasciata sequence (GenBank AY426339), scale bar represents substitutions per site. Accession numbers for sequence generated in this study are given in Additional file 2: Table S4. 
for the repetitive region (Figure 4). Unlike the repetitive region PCR, direct sequencing of terminal region PCR products resulted in extensive numbers of multiple peaks and in some cases, poor sequencing reads due to length differences. Thus, all terminal region PCR products were cloned, a total of 385 amplicons were sequenced, and AcSp1 variants were diagnosed. Each variant was supported by at least two amplicons with sequences that had greater than 95\% identical bases (Figure 4; see Methods). Unlike the repetitive region sequences, which showed no intra-specific or allelic variation, all terminal region amplifications were heterogeneous.

The number of variants characterized was surprising because all of the individual spiders surveyed were found to have more than two terminal region variants, indicating that these Argiope species must have multiple AcSp1 encoding loci. Argiope spiders are not known to be polyploid, thus multiple gene copies per genome is the only explanation for more than two $\mathrm{N}$ - or $\mathrm{C}$ - terminal region variants in a single individual. For example, we found seven $\mathrm{C}$-terminal variants in one $A$. argentata, suggesting at least four AcSp1 gene copies (Figure 4C). Each $A$. trifasciata individual possessed a minimum of seven $\mathrm{N}$ or C-terminal variants, again indicating at least four gene copies (Figure 4B, C). Likewise, an A. aurantia individual possessed six $\mathrm{N}$-terminal variants but only two C-terminal variants (Figure 4B, C). The smaller number of C-terminal variants could be explained by lack of variation in the $\mathrm{C}$-terminal region or by incomplete sampling of variants by PCR survey.

ML analysis of sequences from the PCR survey shows that the branch lengths in the repetitive region (Figure 4A) are shorter than the branch lengths of the terminal region trees (Figure 4B, C). The majority of $\mathrm{N}$ - and C-terminal variants cluster into well-supported, species-specific groups, and intra-specific branch lengths are very short compared to inter-specific branch lengths (Figure 4B, C). One exception is $A$. argentata $\mathrm{C}$-terminal coding region variant $\mathrm{V} 1$, which forms a weakly supported group with A. trifasciata and A. aurantia C-terminal coding region variants (Figure 4C). Given the weak clade support, this variant is probably an outlier that is not as homogenized as the other $A$. argentata variants.

The shorter branch lengths of the repetitive region variants tree compared to those of the $\mathrm{N}$ - and C-terminal region trees suggest that the repetitive region is the most conserved araneid AcSp1 region (Figure 4). Yet, comparison of the average ratio of non-synonymous to synonymous substitution rates $(\mathrm{dN} / \mathrm{dS})$ across codons implies that the $\mathrm{N}$-terminal region has been subject to slightly stronger purifying selection than the repetitive and C-terminal regions $(0.20$ vs. 0.30 and $0.42 \mathrm{dN} / \mathrm{dS}$, respectively). $\mathrm{dN} / \mathrm{dS}$ estimations, however, assume independence of sites and thus are confounded by factors such as concerted evolution and recombination. The full-length $A a r_{-} A c S p 1$ and other $A c S p 1$ provide extensive evidence that the repetitive region units are most likely not evolving independently from each other (Figure 3A; $[1,19])$. Thus concerted evolution and purifying selection both must play a role in the near-perfect homogeneity of Argiope AcSp1 iterated repeats. Recombination can also affect tests of selection [35]. Because we could not conclusively determine the exact number of loci within an individual or assign alleles to specific loci, we were unable to ascertain recombination between loci. Subsequent analyses with additional data could address the impact of recombination on $\mathrm{dN} / \mathrm{dS}$ estimates.

Previous work with $A c S p 1$ sequences did not find evidence for multiple loci $[1,19]$. The lack of variation among A. trifasciata AcSp1 cDNA clones [1] may be due to overexpression or preferential cloning of one variant and thus its preponderance in the characterized cDNAs. Alternatively, consistent depletion of aciniform silk may be required to stimulate transcription of multiple AcSp1 loci. This hypothesis is supported by a significant increase in aciniform-silk dependent web-decorating behavior in three species of Argiope in response to a two-week period of aciniform silk depletion [4]. Future work could focus on comparing the number of $A c S p 1$ variants expressed by spiders consistently depleted of aciniform silk versus that from spiders that are not depleted.

Survey of individual $L$. hesperus genomes also did not find AcSp1 variants [19]. However, the detection of multiple AcSp1 loci in Argiope but not Latrodectus is consistent with the hypothesis that Argiope spiders maintain multiple gene copies as a strategy for efficiently producing large amounts of protein. In contrast with Argiope spiders, Latrodectus spiders use markedly fewer strands of aciniform silk during prey capture [3] and do not make stabilimenta. Increased AcSp1 copy number in Argiope spiders may therefore be a strategy for increasing protein production [36]. Because spidroins are costly, highly expressed proteins [37,38], resource abundance in the form of prey availability may also stimulate aciniform spidroin production in Argiope to prepare for resource scarcity $[39,40]$. Precedent for this strategy exists. In the bacteria Escherichia coli, multiple copies of rRNA operons provide a competitive advantage by enabling increased growth rates and decreased cell division lag time in environments where resources fluctuate rapidly $[41,42]$.

Previous research has found variants for other spidroins [43-46], and that the dragline spidroin MaSp1 is encoded by multiple loci in several species $[47,48]$. Unlike $A$. argentata $A c S p 1$ variants, the C-terminal coding region of $L$. hesperus MaSp1 is nearly identical across loci [47]. This difference could indicate functional constraints on the C-terminal coding region of MaSp1 that either differ from or are not acting on AcSp1. A comparison of 
structural component predictions from the amino acid sequences of terminal regions across different spidroins would greatly inform our understanding of the contribution of the terminal regions to the evolution of different spider silk types.

\section{Conclusions}

The highly similar iterated repeat array of our complete Argiope argentata AcSp1 gene combined with sequence conservation of functionally important regions of individual repeats supports a hypothesis of concerted evolution and functional constraints acting together to homogenize Aar_AcSp1 repeats. In addition, several terminal region variants per individual Argiope genome indicate multiple Argiope AcSp1 loci. Across AcSp1 loci within an individual, we found homogenization of the repetitive region, but variation at the terminal coding regions. We also found evidence for stronger purifying selection in the $\mathrm{N}$-terminal region versus the repetitive or C-terminal region, suggesting that the $\mathrm{N}$-terminal region is the most constrained portion of the aciniform spidroin. The maintenance of multiple copies of AcSp1 in Argiope genomes underscores the importance of aciniform silk in Argiope ecology and evolution. Indeed, variation between individuals and multiple gene copies within individuals could provide a method for the rapid synthesis of aciniform silk in this genus, and may represent the early stages of the differentiation that led to the extraordinary sequence and functional diversity of spider silks.

\section{Methods}

Isolation and sequencing of AcSp1 containing BAC clone

A bacterial artificial chromosome (BAC) library was constructed by Rx BioSciences (Rockville, MD) with Argiope argentata genomic DNA inserted into $\mathrm{pCC} 1 \mathrm{BAC}$ vector (Epicentre, Madison, WI). Colony pools were PCR screened for $A c S p 1$ with primers designed from the repetitive region of Argiope trifasciata AcSp1 (Additional file 2: Table S1), resulting in one positive clone. The positive clone was restriction enzyme digested and a $\sim 17 \mathrm{~kb}$ Hind III fragment of the full insert was found to contain the complete AcSp1 gene.

The $17 \mathrm{~kb}$ fragment was gel purified with the S.N.A.P. UV-Free Gel Purification Kit (Invitrogen, Carlsbad, CA), ligated into HindIII digested $\mathrm{pZErO}^{\mathrm{Tm}}-2$ plasmid (Invitrogen), and transformed into TOP10 cells (Invitrogen). Seven plasmid clones with the expected insert size and restriction enzyme digest patterns were end-sequenced with M13 and Sp6 primers to identify orientation of the inserts. Two clones (one of each insert orientation) were tripledigested with SpeI, XbaI, and XhoI and the fragments were gel-purified. The two clones were also singledigested with BamHI and the largest fragment from each digest $(5.5 \mathrm{~kb}$ or $5.9 \mathrm{~kb}$, composed of the vector and either a $2.2 \mathrm{~kb}$ or $2.6 \mathrm{~kb}$ insert fragment) was gelpurified and re-circularized to produce subclones. The triple digest produced a $12.4 \mathrm{~kb}$ SpeI/XbaI fragment that was gel-purified and subcloned into SpeI digested pZErO-2 plasmid. End-sequencing the subclones revealed that the 2.6, 12.4, and $2.2 \mathrm{~kb}$ inserts corresponded to the AcSp1 N-terminal, repetitive, and C-terminal encoding regions, respectively. The 2.6 and $2.2 \mathrm{~kb}$ fragments were sequenced in their entirety using primer walking (Additional file 2: Table S1). Because the $12.4 \mathrm{~kb}$ fragment contained repetitive nucleotide sequence, primer walking was not feasible. Instead, the $12.4 \mathrm{~kb}$ fragment was bidirectionally sequenced in its entirety using the transposon-based EZ-Tn5 < TET-1 > Insertion Kit (Epicentre Biotechnologies). The complete contig of the $17 \mathrm{~kb}$ genomic fragment was manually assembled with Sequencher 4.5 (Gene Codes, Ann Arbor, MI). An additional $1 \mathrm{~kb}$ of genomic sequence immediately adjacent to the 3' end of the $17 \mathrm{~kb}$ fragment was determined by primer walking (Additional file 2: Table S1) using the original BAC clone as template DNA. The complete Argiope argentata AcSp1 gene was uploaded to GenBank with the accession number KJ206620.

\section{Inter- and Intraspecific sampling of $\mathrm{N}-$, repetitive, and $\mathrm{C}$-terminal coding region fragments}

Genomic DNA was extracted from single legs removed from four $A$. argentata, one $A$. aurantia, and two $A$. trifasciata individuals using the DNeasy Blood \& Tissue Kit (Qiagen, Valencia, CA). N-terminal, repetitive, and C-terminal encoding fragments of $A c S p 1$ were PCR amplified using primers designed from the A. argentata AcSp1 complete gene (Additional file 2: Table S1).

PCR products of the expected size were purified using the AccuPrep Gel Purification Kit (Bioneer Inc., Alameda, CA). Products were directly sequenced. If a chromatograph had overlapping peaks, indicative of heterogeneous amplification, then the product was ligated into pJET 1.2 plasmid (ThermoScientific) and transformed into TOP10 cells. Individual colonies were PCR amplified using pJET1.2 Forward and Reverse sequencing primers. Inserts of the expected size were gel purified and sequenced. If one variant was highly abundant, then additional colonies were PCR amplified and digested with restriction enzymes to identify the abundant variants. The remaining undigested PCR products containing the rare variant were purified and sequenced.

\section{Diagnosing variants}

Nucleotide sequences from the PCR fragments from each species were aligned as described below. For variant diagnosis, single nucleotide polymorphisms (SNPs) that were present in only one individual clone were 
attributed to Taq polymerase error and that SNP was ignored. If a sequence had a pattern of polymorphism that was not present in at least one other clone, the sequence was discarded. Neighbor joining trees were then used to visualize highly similar sequences. Clusters that had greater than $95 \%$ identical sites were considered a variant group. With the exception of the cluster for $A$. trifasciata C-terminal coding variant 14 (95.2\% identical sites), all clusters had greater than $98 \%$ identical bases. Clustered sequences were extracted and aligned to derive the majority rule consensus for that variant. Each variant is therefore supported by at least two sequences. Variants were uploaded to GenBank with accession numbers KJ206570-KJ206619.

\section{Phylogenetic analyses}

The conserved spidroin $\mathrm{N}$ - and C-terminal regions from the complete $A$. argentata AcSp1 were aligned to 29 published spidroins that also have both $\mathrm{N}$ - and C-terminal regions (accession numbers in Additional file 2: Table S2) using ClustalW [49] implemented in Geneious v6.1.6 (Biomatters Ltd., Auckland, NZ). The N- and C-terminal regions were separately aligned with default settings and the alignments were adjusted by eye. The aa alignments dictated nucleotide alignments. $\mathrm{N}$ - and $\mathrm{C}$-terminal encoding region alignments were concatenated for phylogenetic analyses of spidroin paralogs (Additional file 1: Figure S2). Despite potential recombination and convergence in the $\mathrm{N}$ - and C-terminal encoding regions, previous research found no conflict between the strongly supported nodes between separate $\mathrm{N}$ - and $\mathrm{C}$-terminal trees and that concatenation of the terminal regions provides greater evolutionary resolution [12].

The 20 repeat units from the complete $A$. argentata AcSp1 repetitive region were divided into individual files and aligned as above with individual repeat units from published araneid AcSp1 sequences (Additional file 1: Figure S3, accession numbers in Additional file 2: Table S3). Alignments for the $\mathrm{N}$ - and C-terminal encoding sequences obtained from the PCR survey of individual genomes were created as above using diagnosed variants. Repetitive region alignments from the PCR survey also used the above method (alignments in Additional file 1: Figures S4-S6).

For each nucleotide alignment, bootstrap and maximum likelihood (ML) searches for optimal trees were simultaneously conducted over 5,000 replicates using RAxML 7.2.8 with the GTRGAMMA model [50,51] through the CIPRES webserver [52]. As implemented through CIPRES, RAxML has two substitution models: GTRGAMMA and GTRCAT. GTRGAMMA is considered more thorough [50-52]. Accession numbers given in Additional file 2: Tables S2-S4.

\section{Selection analyses}

Estimates of the number of nonsynonymous substitutions per nonsynonymous sites $(\mathrm{dN})$ and synonymous substitutions per synonymous sites $(\mathrm{dS})$ were produced for three AcSp1 nucleotide alignments using MEGA5 [53]: $\mathrm{N}$-terminal encoding variants (Figure 4B; Additional file 1: Figure S4), iterated repeats (Figure 3; Additional file 1: Figure S3), and C-terminal encoding variants (Figure 4C; Additional file 1: Figure S5). CodonTest [54] implemented through the Datamonkey webserver $[55,56]$ indicated the Felsenstein 1981 (F81) [57] model of codon substitution as the best-fit for all analyzed datasets. $\mathrm{dN} / \mathrm{dS}$ ratios less than, equal to, or greater than 1 were interpreted as purifying selection, neutrality, or positive selection, respectively.

\section{Availability of supporting data}

All sequences generated in this study are deposited in GenBank (KJ206570-KJ206620). Alignments used in $\mathrm{ML}$ analyses are available shown in the additional files. Alignments and the corresponding trees for this study are available at TreeBASE (http://purl.org/phylo/treebase/ phylows/study/TB2:S15355).

\section{Additional files}

\footnotetext{
Additional file 1: Figure S1. Nucleotide alignment of the 20 repeat units (Aar_R) from the complete A. argentata AcSp1 repetitive region. Alignment position numbers shown in increments of ten. Frame 1 translation is shown under nucleotide sequences. Alignment prepared with Geneious v6.1.6 (Biomatters Ltd., Auckland, NZ). Figure S2. Nucleotide alignment of 30 concatenated AcSp1 N- and C- terminal coding regions. Alignment position numbers shown in increments of ten. Frame 1 translation is shown under nucleotide sequences. Alignment positions 1-510 encompass the N-terminal coding region, 511-840 the C-terminal coding region. Alignment prepared with Geneious v6.1.6 (Biomatters Ltd., Auckland, NZ) and available on TreeBASE (http:/purl.org/phylo/treebase/phylows/study/ TB2:S15355). Abbreviatons and accession numbers in Additional file 2: Table S2. Figure S3. Nucleotide alignment of Araneidae AcSp1 iterated repeats. Alignment position numbers shown in increments of ten. Frame 1 translation is shown under nucleotide sequences. Alignment prepared with Geneious v6.1.6 (Biomatters Ltd., Auckland, NZ) and available on TreeBASE (http://purl.org/phylo/treebase/phylows/study/TB2:S15355). Abbreviations and accession numbers in Additional file 2: Table S3. Figure S4. Nucleotide alignment of AcSp $1 \mathrm{~N}$-terminal coding variants from PCR survey of individual Argiope genomes. Nucleotide alignment in FASTA format. Alignment available on TreeBASE (http://purl.org/ phylo/treebase/phylows/study/TB2:S15355). Abbreviations: A. argentata (Aarg), A. aurantia (Aau), and A. trifasciata (At). Figure S5. Nucleotide alignment of ACSp1 C-terminal coding variants from PCR survey of individual Argiope genomes. Nucleotide alignment in FASTA format. Alignment available on TreeBASE (http://purl.org/phylo/treebase/ phylows/study/TB2:S15355). Abbreviations: A. argentata (Aarg), A. aurantia (Aau), A. trifasciata (At), and Araneus ventricous (Av). Figure S6. Nucleotide alignment of AcSp 1 repeat region from PCR survey of individual Argiope genomes. Nucleotide alignment in FASTA format. Alignment available on TreeBASE (http://purl.org/phylo/treebase/phylows/study/TB2:S15355). Abbreviations: A. argentata (Aarg), A. aurantia (Aau), A. trifasciata (At), and Araneus ventricosus (Av)

Additional file 2: Table S1. Primers used for full-length Aar_AcSp1 sequencing and targeted amplification of $\mathrm{N}$-terminal, repetitive, and $\mathrm{C}$-terminal coding regions. The name and sequence of primers designed for
} 
primer walking during $\mathrm{BAC}$ clone sequencing and primers designed to amplify $\mathrm{N}$-terminal, repetitive, and C-terminal regions of Aar_AcSpl are given. Table S2. Accession numbers of spidroin sequences used for maximum likelihood analysis of terminal regions (Figure 2). Spidroin name is the name used in this manuscript. Species is the spider species that corresponds to the $\mathrm{N}$ - or C-terminal accession number. If a full length gene was used, only one accession number appears. Table S3. Accession numbers for sequences used in maximum likelihood analyses of iterated repeats (Figure 3). Spidroin name is the name used in this manuscript. Species is the spider species that corresponds to the $\mathrm{N}$ - or $\mathbf{C}$-terminal accession number. Table $\mathbf{S} 4$. Accession numbers for AcSpl sequences generated in this study and used in maximum likelihood analyses of repeat region and $\mathrm{N}$ - and C-terminal encoding variants (Figure 4). GenBank abbreviations, species, and accession number are given. Table S5. Predicted amino acid composition and codon usage of the coding region of Aar_AcSp1. The percentage Aar_AcSpl composed of each amino acid and percentage of each codon used for each amino acid. Table S6. Overall and putative helical region pairwise identities of $A$. trifasciata consensus AcSp1 repeat aligned to consensus AcSp1 repeat subparts of $L$. hesperus and $U$. diversus. Consensus repeat sequences from each subpart of AcSp1 repeats from $L$. hesperus and $U$. diversus were aligned to a consensus repeat from A. trifasciata. Overall pairwise percent identity and percent identity shown for each of six helical regions as predicted by Xu et al [32].

\section{Abbreviations}

aa: amino acid; BAC: bacterial artificial chromosome; bp: base pairs; cDNA: complementary deoxyribonucleic acid; C-terminal: carboxy terminal; $\mathrm{dN}$ : non-synonymous substitution rate; dS: synonymous substitution rate; kb: kilobases; kDa: kilodaltons; ML: maximum likelihood; MYA: million years ago; NMR: nuclear magnetic resonance; $\mathrm{N}$-terminal: amino-terminal; PCR: polymerase chain reaction; rDNA: DNA sequence that codes for ribosomal RNA; rRNA: ribosomal ribonucleic acid; SNP: single nucleotide polymorphism.

\section{Competing interests}

The authors declare that they have no competing interests.

\section{Authors' contributions}

RCC collected and analyzed data and drafted the manuscript, YZ, JW, RA, and KA collected data. NA contributed to conception and design of the study. CYH conceived the study, collected data, and drafted the manuscript. All authors reviewed and approved the final manuscript.

\section{Acknowledgements}

We thank Fanny Chan and Marissa Panyawai for help with lab work. Sandra Correa-Garwhal, John Gatesy, James Starrett, and two anonymous reviewers improved the manuscript. Todd Blackledge provided A. aurantia specimens. This work was funded by U.S. Army Research Office grant W911NF-11-1-0299.

\section{Author details}

'Department of Biology, University of California, 900 University Avenue, Riverside 92507, Riverside, CA, USA. ${ }^{2}$ Faculty of Life Science, Liaoning University, Province Shenyang, Liaoning 110036, China. ${ }^{3}$ Department of Biology, Washington and Lee University, 204 W. Washington St., Lexington, VA 24450, USA.

Received: 25 October 2013 Accepted: 14 February 2014 Published: 20 February 2014

\section{References}

1. Hayashi CY, Blackledge TA, Lewis RV: Molecular and mechanical characterization of aciniform silk: uniformity of iterated sequence modules in a novel member of the spider silk fibroin gene family. Mol Biol Evol 2004, 21:1950-1959.

2. Vasanthavada K, Hu X, Falick A, Mattina CL, Moore A, Jones P, Yee R, Reza R, Tuton T, Vierra C: Aciniform spidroin, a constituent of egg case sacs and wrapping silk fibers from the black widow spider Latrodectus hesperus. J Biol Chem 2007, 282:35088-35097.
3. Griswold CE, Coddington JA, Hormiga G, Scharff N: Phylogeny of the orb-web building spiders (Araneae, Orbiculariae: Deinopoidea, Araneoidea). Zool J Linn Soc-Lond 1998, 123:1-99.

4. Walter A, Elgar MA, Bliss P, Moritz RFA: Wrap attack activates web-decorating behavior in Argiope spiders. Behav Ecol 2008, 19:799-804.

5. Walter A, Elgar MA: The evolution of novel animal signals: silk decorations as a model system. Biol Rev 2012, 87:686-700.

6. Herberstein $\mathrm{ME}$, Craig $\mathrm{CL}$, Coddington JA, Elgar MA: The function significance of silk decorations of orb-web spiders: a critical review of the empirical evidence. Biol Rev Camb Philos Soc 2000, 75:649-669.

7. Blackledge TA, Hayashi CY: Silken toolkits: biomechanics of silk fibers spun by the orb web spider Argiope argentata (Fabricius 1775). J Exp Biol 2006, 209:2452-2461.

8. Hinman MB, Lewis RV: Isolation of a clone encoding a second dragline silk fibroin. Nephila clavipes dragline silk is a two-protein fiber. J Biol Chem 1992, 267:19320-19324.

9. Gatesy J, Hayashi C, Motriuk D, Woods J, Lewis R: Extreme diversity, conservation, and convergence of spider silk fibroin sequences. Science 2001, 291:2603-2605.

10. Garb JE, DiMauro T, Lewis RV, Hayashi CY: Expansion and intragenic homogenization of spider silk genes since the Triassic: evidence from Mygalomorphae (tarantulas and their kin) spidroins. Mol Biol Evol 2007, 24:2454-2464

11. Rising A, Hjälm G, Engström W, Johansson J: N-terminal nonrepetitive domain common to dragline, flagelliform, and cylindriform spider silk proteins. Biomacromolecules 2006, 7:3120-3124.

12. Garb JE, Ayoub NA, Hayashi CY: Untangling spider silk evolution with spidroin terminal domains. BMC Evol Biol 2010, 10:243-259.

13. $\mathrm{Xu} M$, Lewis RV: Structure of a protein superfiber: spider dragline silk. Proc Natl Acad Sci U S A 1990, 87:7120-7124.

14. Jenkins JE, Creager MS, Butler EB, Lewis RV, Yarger JL, Holland GP: Solid-state NMR evidence for elastin-like beta-turn structure in spider dragline silk. Chem Commun (Camb) 2010, 46:6714-6716.

15. Xu L, Tremblay M-L, Orrell KE, Leclerc J, Meng Q, Liu X-Q, Rainey JK: Nanoparticle self-assembly by a highly stable recombinant spider wrapping silk protein subunit. FEBS Lett 2013, 587:3273-3280.

16. Beckwitt R, Arcidiacono S: Sequence conservation in the C-terminal region of spider silk proteins (Spidroin) from Nephila clavipes (Tetragnathidae) and Araneus bicentenarius (Araneidae). J Biol Chem 1994, 269:6661-6663.

17. Sponner A, Unger $E$, Grosse F, Weisshart $K$ : Conserved C-termini of spidroins are secreted by the major ampullate glands and retained in the silk thread. Biomacromolecules 2004, 5:840-845.

18. Hu X, Kohler K, Falick AM, Moore AMF, Jones PR, Vierra C: Spider egg case core fibers: trimeric complexes assembled from TuSp1, ECP-1, and ECP-2. Biochemistry 2006, 45:3506-3516.

19. Ayoub NA, Garb JE, Kuelbs A, Hayashi CY: Ancient properties of spider silks revealed by the complete gene sequence of the prey-wrapping silk protein (AcSp1). Mol Biol Evol 2012, 30:589-601.

20. Beckwitt R, Arcidiacono S, Stote R: Evolution of repetitive proteins: spider silks from Nephila clavipes (Tetragnathidae) and Araneus bicentenarius (Araneidae). Insect Biochem Mol Biol 1998, 28:121-130.

21. Ganley ARD, Kobayashi T: Highly efficient concerted evolution in the ribosomal DNA repeats: Total rDNA repeat variation revealed by wholegenome shotgun sequence data. Genome Res 2007, 17:184-191.

22. Swanson WJ, Vacquier VD: Concerted evolution in an egg receptor for a rapidly evolving abalone sperm protein. Science 1998, 281:710-712.

23. Hayashi $C Y$, Lewis RV: Molecular architecture and evolution of a modular spider silk protein gene. Science 2000, 287:1477-1479.

24. Wang S, Huang W, Yang D: NMR structure note: repetitive domain of aciniform spidroin 1 from Nephila antipodiana. J Biomol NMR 2012, 54:415-420

25. Xu L, Tremblay ML, Meng Q, Liu XQ: $1 \mathrm{H}, 13 \mathrm{C}$ and $15 \mathrm{~N}$ NMR assignments of the aciniform spidroin (AcSp1) repetitive domain of Argiope trifasciata wrapping silk. Biomol NMR Assigm 2012, 6:147-151.

26. Hayashi CY, Lewis RV: Evidence from flagelliform silk cDNA for the structural basis of elasticity and modular nature of spider silks. J Mol Biol 1998, 275:773-784.

27. Ayoub NA, Hayashi CY: Spiders (Araneae). In The Timetree of Life. Edited by Hedges SB, Kumar S. Oxford University Press; 2009:255-259.

28. Tian M, Lewis RV: Molecular characterization and evolutionary study of spider Tubuliform (eggcase) silk protein. Biochemistry 2005, 44:8006-8012. 
29. McAllister BF, Werren $\mathrm{JH}$ : Evolution of tandemly repeated sequences: what happens at the end of an array? J Mol Evol 1999, 48:469-481.

30. Hayashi CY, Lewis RV: Spider flagelliform silk: lessons in protein design, gene structure, and molecular evolution. Bioessays 2001, 23:750-756.

31. Su YC, Chang YH, Smith D, Zhu MS, Kuntner M, Tso IM: Biogeography and speciation patterns of the golden orb spider genus Nephila (Araneae:Nephilidae) in Asia. Zool Sci 2011, 28(1):47-55.

32. Elices M, Plaza GR, Arnedo MA, Perez-Riquerio J, Torres FG, Guinea GV: Mechanical behavior of silk during the evolution of orb-web spinning spiders. Biomacromolecules 2009, 10(7):1904-1910.

33. Lefèvre $T$, Boudreault $S$, Cloutier $C$, Pézolet M: Diversity of molecular transformations involved in the formation of spider silks. J Mol Biol 2011 405:238-253.

34. Gosline JM, Guerette PA, Ortlepp CS, Savage KN: The mechanical design of spider silks: from fibroin sequence to mechanical function. J Exp Biol 1999, 202:3295-3303.

35. Anisimova M, Nielsen $R$, Yang Z: Effect of recombination on the accuracy of the likelihood method for detecting positive selection at amino acid sites. Genetics 2002, 164:1229-1236.

36. Ohno S: Evolution by Gene Duplication. New York: Springer-Verlag; 1970.

37. Guehrs K-H, Schlott B, Grosse F, Weisshart K: Environmental conditions impinge on dragline silk protein composition. Insect Mol Biol 2008, 17:553-564.

38. Blamires SJ, Wu C-L, Tso I-M: Variation in protein intake induces variation in spider silk expression. PLOS ONE 2012, 7:e31626.

39. Tso IM: The effect of food and silk reserve manipulation on decoration-building of Argiope aetheroides. Behaviour 2004, 141(5):603-616

40. Craig CL, Wolf SG, Davis JLD, Hauber ME, Maas JL: Signal polymorphism in the web-decorating spider Argiope argentata is correlated with reduced survivorship and the presence of stingless bees, its primary prey. Evolution 2001, 55(5):986-993.

41. Condon C, Liveris D, Squires C, Schwartz I, Squires CL: rRNA operon multiplicity in Escherichia coli and the physiological implications of rrn inactivation. J Bacteriol 1995, 177:4152-4156.

42. Stevenson BS, Schmidt TM: Life history implications of rRNA gene copy number in Escherichia coli. Appl Environ Microb 2004, 70:6670-6677.

43. Guerette PA, Ginzinger DG, Weber BH, Gosline JM: Silk properties determined by gland-specific expression of a spider fibroin gene family. Science 1996, 272:112-115.

44. Colgin MA, Lewis RV: Spider minor ampullate silk proteins contain new repetitive sequences and highly conserved non-silk-like "spacer regions". Protein Sci 1998, 7:667-672.

45. Garb JE, DiMauro T, Vo V, Hayashi CY: Silk genes support the single origin of orb webs. Science 2006, 312:1762.

46. Higgins LE, White S, Nuñez-Farfán J, Vargas J: Patterns of variation among distinct alleles of the Flag silk gene from Nephila clavipes. Int J Biol Macromol 2007, 40:201-216.

47. Ayoub NA, Hayashi CY: Multiple recombining loci encode MaSp1, the primary constituent of dragline silk, in widow spiders (Latrodectus: Theridiidae). Mol Biol Evol 2008, 25:277-286.

48. Gaines WA IV, Marcotte WR Jr: Identification and characterization of multiple Spidroin 1 genes encoding major ampullate silk proteins in Nephila clavipes. Insect Mol Biol 2008, 17:465-474.

49. Thompson JD, Higgins DG, Gibson TJ: CLUSTAL W: improving the sensitivity of progressive multiple sequence alignment through sequence weighting, position-specific gap penalties and weight matrix choice. Nucleic Acids Res 1994, 22:4673-4680

50. Stamatakis A: RAxML-VI-HPC: maximum likelihood-based phylogenetic analyses with thousands of taxa and mixed models. Bioinformatics 2006, 22:2688-2690

51. Stamatakis A, Hoover P, Rougemont J: A rapid bootstrap algorithm for the RAxML Web servers. Syst Biol 2008, 57:758-771.

52. Miller MA, Pfeiffer W, Schwartz T: The CIPRES science gateway. In Proceedings of the Gateway Computing Environments Workshop (GCE). New Orleans, LA: ACM Press; 2010:1-8.

53. Tamura K, Peterson D, Peterson N, Stecher G, Nei M, Kumar S: MEGA5: molecular evolutionary genetics analysis using maximum likelihood, evolutionary distance, and maximum parsimony methods. Mol Biol Evol 2011, 28(10):2731-2739.
54. Delport W, Scheffler K, Botha G, Gravenor MB, Muse SV, Pond SLK: CodonTest: modeling amino acid substitution preferences in coding sequences. PLoS Comput Biol 2010, 6(8):e1000885.

55. Pond SLK, Frost SD: Datamonkey: rapid detection of selective pressure on individual sites of codon alignments. Bioinformatics 2005, 21(10):2531-2533.

56. Delport W, Poon AF, Frost SD, Pond SLK: Datamonkey 2010: a suite of phylogenetic analysis tools for evolutionary biology. Bioinformatics 2010, 26(19):2455-2457.

57. Felsenstein J: Evolutionary trees from DNA sequences: a maximum likelihood approach. J Mol Evol 1981, 17:368-376.

doi:10.1186/1471-2148-14-31

Cite this article as: Chaw et al:: Intragenic homogenization and multiple copies of prey-wrapping silk genes in Argiope garden spiders. BMC Evolutionary Biology 2014 14:31.

\section{Submit your next manuscript to BioMed Central and take full advantage of:}

- Convenient online submission

- Thorough peer review

- No space constraints or color figure charges

- Immediate publication on acceptance

- Inclusion in PubMed, CAS, Scopus and Google Scholar

- Research which is freely available for redistribution 